\title{
Solar Neutrinos: Models, Observations and New Opportunities
}

\author{
W. C. Haxton ${ }^{\mathrm{A}}$ \\ A Institute for Nuclear Theory and Department of Physics, University of Washington, \\ Seattle, WA 98195, USA. Email: haxton@ phys.washington.edu
}

Received 2007 October 10, accepted 2008 March 8

\begin{abstract}
I discuss the development and resolution of the solar neutrino problem, as well as opportunities now open to us to extend our knowledge of main-sequence stellar evolution and neutrino astrophysics.
\end{abstract}

Keywords: neutrinos — stars: evolution

\section{Introduction: A Brief History of the Solar Neutrino Problem}

This paper is based on a talk given at the Caltech conference $^{1}$ 'Nuclear Astrophysics 1957-2007: Beyond the First 50 Years', July 23-27 2007, which focussed on the state of nuclear astrophysics fifty years after the seminal papers of Burbidge, Burbidge, Fowler and Hoyle (Burbidge et al. 1957) and Cameron (1957). The quest to measure solar neutrinos, and later to resolve the solar neutrino problem, began in the early days of nuclear astrophysics, with the first efforts to understand proton burning in main sequence stars. I would like to review that history, our current understanding of solar neutrinos, and open questions in neutrino physics, and discuss some opportunities for further solar neutrino measurements.

Solar neutrino physics brings together stellar modeling, nuclear reactions, and observation. A key early development was the Holmgren \& Johnston (1959) measurement of the $S$-factor for the pp-chain reaction ${ }^{3} \mathrm{He}(\alpha, \gamma){ }^{7} \mathrm{Be}$, which proved to be surprisingly large. This implied that the Sun could produce some of its energy through the more temperature dependent ppII and ppIII cycles of the pp-chain, elevating the neutrino fluxes expected from ${ }^{7} \mathrm{Be}$ electron capture and ${ }^{8} \mathrm{~B} \beta$-decay (see Figure 1 ). These neutrinos contribute to ground- and excited-state transitions in ${ }^{37} \mathrm{Cl}(v, \mathrm{e}){ }^{37} \mathrm{Ar}$, a reaction for detecting neutrinos that had been proposed by Pontecorvo (1946) and considered by Alvarez (1949), who studied backgrounds that might inhibit, for example, a reactor neutrino experiment. The experiment envisioned by Alvarez — proposed as a test of the distinguishability of the neutrino and antineutrino, prior to the discovery of parity violation - was later conducted by Davis at Savannah River (Bahcall \& Davis 1982; Davis 1955).

The Caltech effort in nuclear astrophysics brought three young researchers, Bahcall, Iben and Sears, together in the summer of 1962. Stimulated in part by the Holmgren and Johnston measurement, they began construction of a solar model to predict the solar core temperature, the most

${ }^{1}$ http: //www.na2007.caltech.edu/

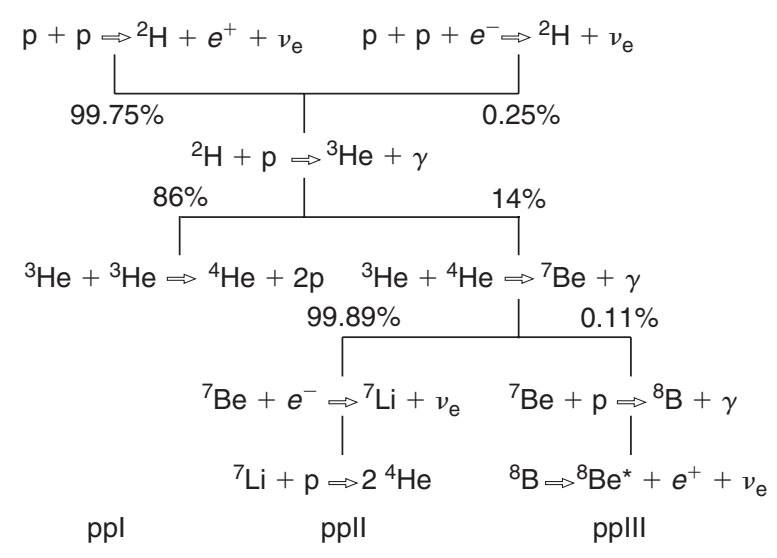

Figure 1 The three cycles of the pp-chain and associated neutrinos. The SSM branching ratios, in percent, are indicated at the three points where pairs of reactions compete.

important parameter governing the competition between the ppI, ppII and ppIII cycles, and to provide the first quantitative estimate of the resulting neutrino fluxes. The Bahcall, Fowler, Iben and Sears model, published in 1963, predicted a counting rate for Davis's proposed 100000 gallon chlorine solar neutrino detector of about one event per day.

A key development occurred in 1963 when, during a seminar by Bahcall at Copenhagen, Mottelson inquired about the importance of neutrino excitation of excited states in ${ }^{37} \mathrm{Ar}$ (Bahcall \& Davis 1982). Bahcall and Barnes (1964) pointed out that a calibration of the excited state contribution to the ${ }^{37} \mathrm{Cl}$ cross section could be made by measuring the delayed protons from the analog $\beta$-decay of ${ }^{37} \mathrm{Ca}$, the isospin mirror of ${ }^{37} \mathrm{Cl}$. Effectively the lifetime of

${ }^{37} \mathrm{Ca}$ would be a test of the elevated cross section predicted on the basis of the excited-state contribution to ${ }^{8} \mathrm{~B}$ neutrino absorption. Subsequent measurements by Hardy and Verrall (1964) and Reeder, Poskanzer and Esterlund (1964) established the importance of the excited-state contribution. (These experiments were later repeated in a manner that was kinematically complete: see Adelberger \& Haxton 1987 for a discussion.) 
Bahcall (1964) and Davis (1964) published companion letters in March 1964 arguing the adequacy and feasibility of a 100000-gallon $\mathrm{Cl}$ experiment to measure solar neutrinos. Excavation of the detector cavity in the Homestake Mine began in summer 1965. The tank was installed and filled by the next year. The first results were announced by Davis, Harmer and Hoffman (1968): an upper bound of $3 \mathrm{SNU}$ ( $1 \mathrm{SNU}=10^{-36}$ captures per $\mathrm{Cl}$ atom per second), below the standard solar model (SSM) prediction of $7.5 \pm 3$ SNU of Bahcall, Bahcall and Shaviv (1968).

This result and associated theoretical work on suggested solutions led to a series of experiments: Gallex (Hampel et al. 1999), GNO (Altmann et al. 2000), SAGE (Abdurashitov et al. 1999a,b), Kamiokande (Hirata et al. 1991), Super-Kamiokande (Hosaka et al. 2006) and the Sudbury Neutrino Observatory (Ahmad et al. 2002). Efforts by the Borexino Collaboration (Arpesella et al. 2007) and KamLAND (Eguchi et al. 2003) to measure the ${ }^{7} \mathrm{Be}$ neutrinos are currently underway or in preparation. These experiments are important as tests of the SSM and of the new neutrino physics that proved to be the source of the solar neutrino problem.

\section{The Standard Solar Model}

The physics assumptions underlying the SSM include:

- Hydrostatic equilibrium For each volume element it is assumed that gravity is balanced by the gas pressure gradient. This requires specification of the electron gas equation of state as a function of temperature, heavy element abundance $Z$ and density. The EOS is very nearly that of an ideal gas.

- Energy transport The Sun has a radiative interior and convective envelope, with the location of the boundary sensitive to the modeling of the radiative opacity. The depth of the convective zone can be determined experimentally, because it influences solar surface acoustic modes (helioseismology).

- Energy generation Solar energy is generated by the conversion of four protons to ${ }^{4} \mathrm{He}$ with the release of about $25 \mathrm{MeV}$, with the pp-chain accounting for nearly 99\% of the reactions (and the CNO cycle the remainder). The main laboratory task has been determining the nuclear cross sections for the various reactions to sufficient precision. Because typical center-of-mass energies in the solar core are $\sim 2 \mathrm{keV}$, in general this requires measuring reactions at higher energies, then using r-matrix or other models to extrapolate the laboratory $S$-factors to threshold.

- Boundary conditions The Sun's age and current luminosity, radius, mass and surface composition are known. While the composition of the solar core at the onset of the main sequence is not known directly, the SSM assumes that the zero-age core metallicity $Z$ can be equated to today's surface value. (Prior to the onset of the main sequence, the proto-Sun is believed to have gone through a fully convective Hayashi phase (Hayashi 1966). Provided that no anomalous deposition of metals occurs after this phase, the radiative core of the zero-age main-sequence Sun would be chemically identical to today's surface, apart from corrections associated with $\mathrm{He}$ and heavy-element diffusion.) As the mass fractions in $\mathrm{H}$, He and heavy elements $Z$ must sum to one, a single additional constraint is needed. This is the solar luminosity: the zero-age $\mathrm{H} / \mathrm{He}$ ratio is adjusted until the correct luminosity is achieved after $4.6 \mathrm{Gyr}$ of evolution.

The nuclear physics efforts on the pp-chain have reach a very high level of sophistication (Haxton, Parker \& Rolfs 2006). Long the most uncertain rate in the pp-chain, the $S$-factor for ${ }^{7} \mathrm{Be}(\mathrm{p}, \gamma)$ has now been determined to an accuracy of $\lesssim 5 \%$

$$
S_{17}(20 \mathrm{keV})=20.6 \pm 0.5 \pm 0.6 \mathrm{eV}-\mathrm{b}
$$

by a series of six 'direct' measurements (Junghans et al. 2003). There have also been 'indirect' measurements involving Coulomb dissociation or heavy-ion reactions. Also notable are the measurements by the LUNA collaboration, working in the low-background environment of Gran Sasso, on ${ }^{3} \mathrm{He}+{ }^{3} \mathrm{He}$. The group succeeded in obtaining data in the solar Gamow peak, thus largely eliminating theoretical uncertainties in the extrapolation of high-energy data to solar energies (Bonetti et al. 1999).

The SSM has evolved over the years. For example, the growing accuracy of helioseismology helped motivate efforts to include the diffusion of He and heavy elements over the solar lifetime. Important checks on the SSM include the predicted depth of the convective zone, which is constrained experimentally by the frequency distribution of low- $l$ acoustic modes. The model that emerges is dynamic: there is a $\sim 44 \%$ luminosity growth over the solar lifetime due to changing core chemistry and thus opacity. The high-energy ${ }^{8} \mathrm{~B}$ neutrino flux is a relatively contemporary phenomenon: it is predicted to grow with a time constant $\tau_{0} \sim 0.9 \mathrm{Gyr}, \phi\left({ }^{8} \mathrm{~B}\right) \sim \phi_{0} e^{-t / \tau_{0}}$. Yet the model remains somewhat limited in scope. As calculations are done in $1 \mathrm{D}$, there is no attempt to model the detailed behavior of the convective zone or of the convective onset of main-sequence burning. Known phenomena such as the depletion of surface Li are presumably connected with such physics.

Solar neutrinos were initially viewed - and remain an important test of the SSM. The three cycles making up the pp-chain (see Figure 1) are tagged by neutrinos: the pp neutrino flux constrains the overall rate of $\mathrm{H}$ burning, while the ${ }^{7} \mathrm{Be}$ and ${ }^{8} \mathrm{~B}$ neutrino fluxes can be used to determine the ppII and ppIII rates, respectively. Because of Coulomb barriers, the competition between the three cycles depends rather sensitively on the solar core temperature. Thus solar neutrino flux measurements, given the quality of current calibrations of the nuclear microphysics, can fix the core temperature to an accuracy of about $1 \%$.

But, by the mid90s, instead of such a temperature determination, an important discrepancy had been confirmed. The combination of the $\mathrm{Cl}$, gallium and Kamioka 


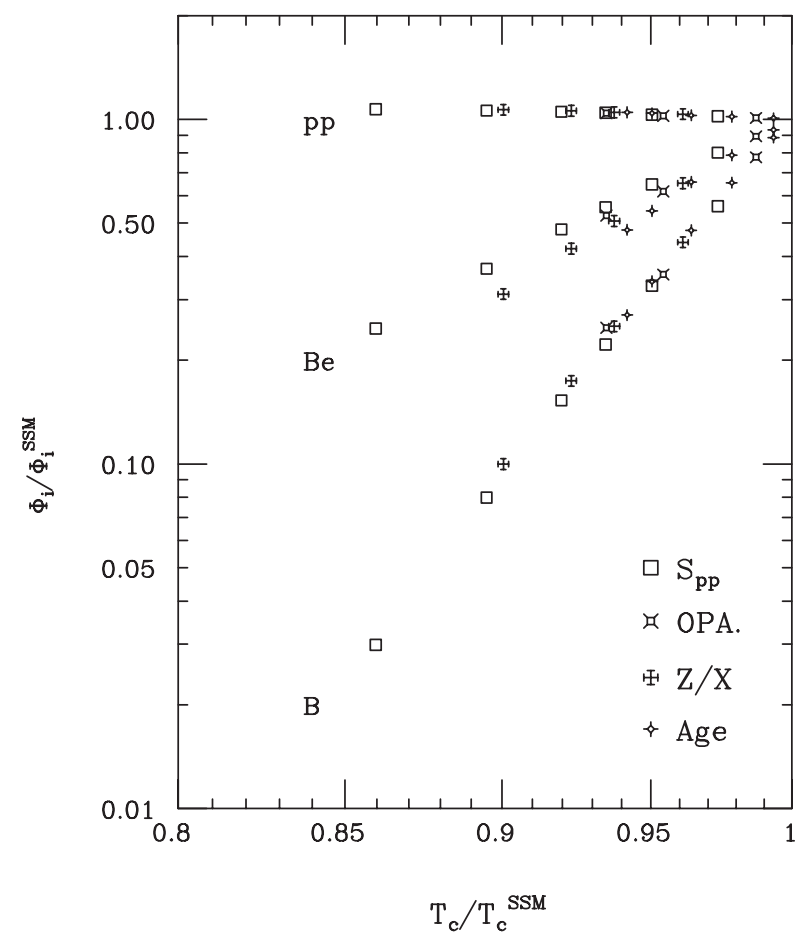

Figure 2 Neutrino fluxes resulting from changes in various SSM parameters, such as the $\mathrm{p}+\mathrm{p} S$-factor, opacity, heavy element abundance and solar age (as indicated by the symbols defined by the key at the lower right). The variations were made far beyond the assigned SSM uncertainties, and thus represent non-SSM extrapolations. The fluxes correlate very well with one parameter, the resulting core temperature $T_{\mathrm{C}}$. This naive $T_{\mathrm{C}}$ dependence was recognised early on to be incompatible with the pattern of fluxes deduced from the $\mathrm{Cl}$, Gallex/SAGE, and Kamioka experiments. From Castellani et al. (1994).

experiments seemed to require (assuming otherwise standard physics):

$$
\begin{aligned}
\phi^{\exp }(\mathrm{pp}) & \sim \phi^{\mathrm{SSM}}(\mathrm{pp}), \\
\phi^{\exp }\left({ }^{7} \mathrm{Be}\right) & \sim 0, \\
\phi^{\exp }\left({ }^{8} \mathrm{~B}\right) & \sim 0.4 \phi^{\mathrm{SSM}}\left({ }^{8} \mathrm{~B}\right) .
\end{aligned}
$$

The difficulty posed by these results can be easily seen.

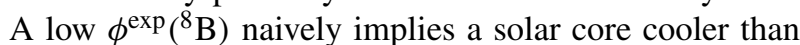
predicted by the SSM. Due to the $\sim T_{\mathrm{C}}^{24}$ dependence of the ${ }^{8} \mathrm{~B}$ neutrinos, a core temperature $\sim 0.96 T_{\mathrm{C}}$ would account for the Kamioka and $\mathrm{Cl}$ results. However, the SSM also predicts that $\phi\left({ }^{7} \mathrm{Be}\right) / \phi\left({ }^{8} \mathrm{~B}\right) \sim T_{\mathrm{C}}^{-14}$. Thus the low value of this ratio, compared to SSM predictions, requires a hotter core. While such an analysis based on a single parameter $T_{\mathrm{C}}$ may seem naive, more detail investigations came to the similar conclusions. For example Castellani et al. (1994, Figure 2) shows that a variety of SSM perturbations yield fluxes that corresponding to expectations based on $T_{\mathrm{C}}$.

\section{Massive Neutrinos}

The standard model (SM) of particle physics has massless neutrinos. But if extended, treated as an effective theory with a dimension-full Majorana mass term (the only dimension-five operator that can be constructed with SM

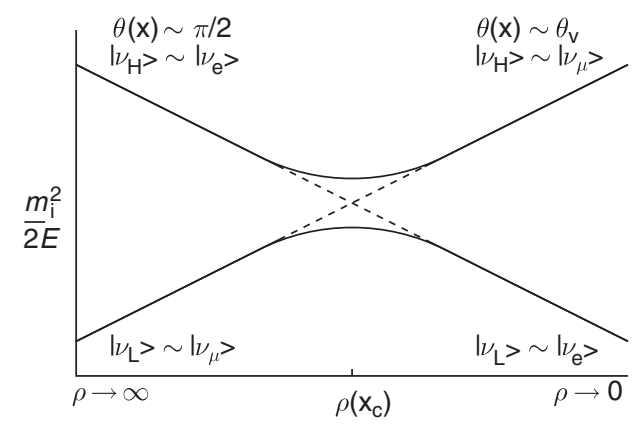

Figure 3 Schematic illustration of the MSW avoided level crossing that may arise because the effective mass of the $v_{\mathrm{e}}$ increases in matter. In the adiabatic limit, a neutrino would follow one of the local mass eigenstate trajectories depicted by the solid lines. A $v_{\mathrm{e}}$ created in the solar core as the heavy mass eigenstate can leave the Sun as the vacuum heavy eigenstate which, if the vacuum mixing angle is small, may be nearly coincident with the $v_{\mu}$.

fields) or enlarged to include the right-handed neutrino field needed for a Dirac mass term, neutrinos would be massive. Massive neutrinos not coincident with their flavor eigenstate counterparts will lead to the phenomenon of neutrino oscillations, as Pontecorvo first pointed out. Furthermore, fascinating new oscillation phenomena can occur because solar neutrinos are created at high density in the solar core, then propagate to low density. Mikheyev and Smirnov (1986) showed that the effective mass neutrinos acquire in matter, a phenomenon first discussed by Wolfstein (1978), could lead to large oscillation probabilities, even for small vacuum mixing angles. Such matter-enhanced neutrino oscillations can be viewed as a level-crossing phenomenon: the local masses of the neutrinos reflect the surrounding electron density, with the electron neutrino becoming heavier at high density. If the solar core density is sufficient to cause a level inversion, then a level crossing will occur somewhere in the Sun as the neutrino propagates outward (Bethe 1986). If that crossing is adiabatic (Haxton 1986; Parke 1986), then strong $v_{\mathrm{e}} \rightarrow v_{\mu}$ conversion will occur, as illustrated in Figure 3. Details of this process are discussed in many places and will not be repeated here.

The early solar neutrino results and the possibility of discovering new particle physics provided the impetus for two important direct-counting experiments, SuperKamiokande (Hosaka et al. 2006) and the Sudbury Neutrino Observatory (SNO, Ahmad et al. 2002). SNO, a Cerenkov detector with an inner vessel containing a kiloton of heavy water surrounded by seven kilotons of light water, was unique in its sensitivity to neutrino flavors. It and Super-Kamiokande both made use of $v_{\mathrm{x}}-e$ elastic scattering (ES)

$$
v_{\mathrm{x}}+e^{-} \rightarrow v_{\mathrm{x}}^{\prime}+e^{-},
$$

a reaction that takes place for both electron and heavyflavor neutrinos, with cross sections roughly in a $6: 1$ ratio. The scattered electrons are forward peaked, allowing the experimenters to cut away backgrounds by correlating signals with the position of the Sun. But SNO also 


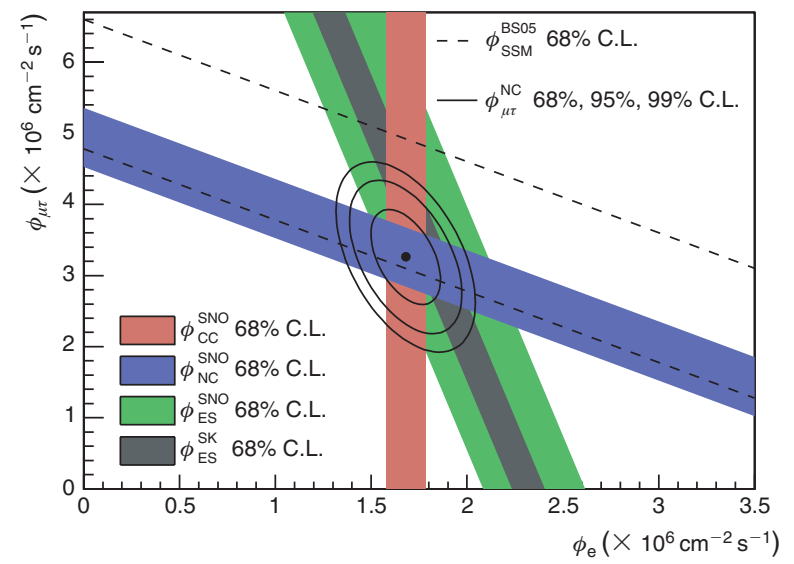

Figure 4 The SNO diagram (Ahmad et al. 2002) showing the region consistent with the $\mathrm{ES}, \mathrm{CC}$, and $\mathrm{NC}$ measurements. Also shown are the Super-Kamiokande ES results (Hosaka et al. 2006) and (designated by dashed lines) the band corresponding to the SSM prediction for the total ${ }^{8} \mathrm{~B}$ neutrino flux.

detected two other reactions, the charged-current (CC) and neutral-current (NC) breakup of deuterium,

$$
\begin{aligned}
& v_{\mathrm{e}}+d \rightarrow p+p+e^{-} \\
& v_{\mathrm{x}}+d \rightarrow v_{\mathrm{x}}^{\prime}+n+p .
\end{aligned}
$$

These reactions are sensitive, respectively, just to electron neutrinos or equally to neutrinos of any flavor. The CC reaction was detected via the scattered electron, which tends to carry off most of the neutrino energy (helpful in reconstructing the neutrino spectrum) but is emitted almost isotropically (so there is little directionality that can be exploited to reduce backgrounds). The signal for the NC reaction is the neutron, which was observed in SNO by $(n, \gamma)$ capture on a Cl-bearing salt added to the detector and, later, in tubular ${ }^{3} \mathrm{He}$ proportional counters that were installed in the detector. SNO's great depth $(\sim 6 \mathrm{~km}$ water equivalent) and clean-room operating standards made such measurements possible, reducing cosmic and environmental radioactivity backgrounds to very low levels.

The SNO results are shown in Figure 4. The bands representing the three neutrino detection channels, with their very different sensitivities to neutrino flavour, converge at an ellipse indicating that approximately two-thirds of the solar neutrinos arrive on earth as heavy-flavor neutrinos. Thus the discrepancy first noted by Davis, whose $\mathrm{Cl}$ detector recorded electron neutrinos only, was not due to an incorrect estimate of the solar neutrino flux, but rather to their partial conversion to other flavors during transit to earth. Indeed, Figure 4 shows that the SSM prediction for the ${ }^{8} \mathrm{~B}$ solar neutrino flux is in good agreement with the flavor-blind NC measurement made in SNO.

\section{Next Steps?}

Thus the question, where does the solar neutrino field go from here? Three important directions are:

- Pursuit of several important open questions about neutrino properties, using both accelerator/reactor and

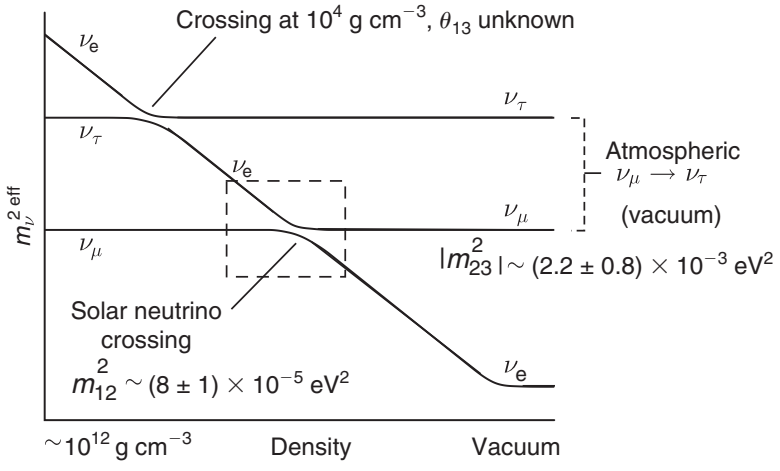

Figure 5 A three-flavor plot showing two MSW crossings, the second of which corresponds to a density of $\sim 10^{4} \mathrm{~g} \mathrm{~cm}^{-3}$, typical of the carbon zone of the progenitor of a Type II supernova. The relevant mixing angle $\theta_{13}$ has not yet been measured.

astrophysical neutrino sources. These questions are important to the modeling of a variety of exotic stellar environments, such as core-collapse supernovae, and to the construction of extensions to the SM that will encompass the new neutrino physics.

- Completing the spectroscopy of pp-chain neutrinos. This includes direct measurements, such as those underway by Borexino (Arpesella et al. 2007) and KamLAND (Eguchi et al. 2003), of the ${ }^{7}$ Be neutrinos, and future experiments to determine the flux and flavor of the dominant solar neutrino source, the pp neutrinos. Existing constraints on the low-energy fluxes come from the radiochemical $\mathrm{Cl}$ and Gallex/GNO/SAGE detectors.

- Measuring the CNO neutrinos. I will argue below that such a measurement is not only important to understanding the Sun - a CNO neutrino measurement would determine directly the solar core metallicity but also to the general theory of main-sequence evolution of massive stars.

\subsection{Neutrino Properties}

Figure 5 illustrates some of the expectations for neutrino physics in stellar environments, assuming a regular (rather than inverted) hierarchy where the two neutrino states participating in solar neutrino mixing are lighter than the third state. The physics discussed in connection with solar neutrinos, the 1-2 level crossing arising from matter effects, is repeated at higher density in a second crossing. The atmospheric $\delta m_{23}^{2} \sim(2.2 \pm 0.8) \times 10^{-3} \mathrm{eV}^{2}$ leads to the expectation that for neutrino energies typical of a supernova $(\sim 10 \mathrm{MeV})$, this crossing would be encountered in the carbon zone of a massive star, where $\rho_{\mathrm{e}} \sim 10^{4} \mathrm{~g} \mathrm{~cm}^{-3}$. However, the relevant mixing angle $\theta_{13}$ is not known: the current upper bound from the Chooz reactor experiment is $\sin ^{2} 2 \theta \lesssim 0.19$, at $\delta m^{2}=2.0 \times 10^{-3} \mathrm{eV}$ (Apollino et al. 1999). This crossing (as well as additional neutrino background effects discussed by George Fuller at 


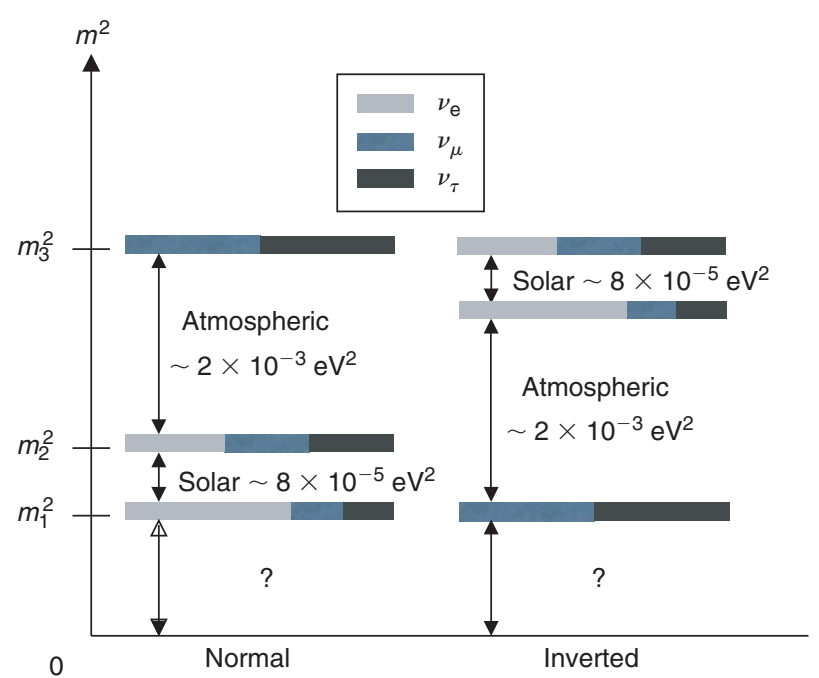

Figure 6 The flavor content, allowable hierarchies, and unknown mass scale of the neutrinos.

this meeting) has the potential to alter energy deposition in a supernova, by causing the exchange of cooler electron neutrinos and hotter heavy-flavor neutrinos. Hotter electron neutrinos increase the neutrino-matter coupling. Such an inversion could produce distinctive signatures in terrestrial detectors with sensitivities to different neutrino flavors. As the crossing is expected to remain adiabatic for mixing angles $\gtrsim 10^{-4}$, this signature could prove very important if $\theta_{13} \lesssim 0.01$, the level that proposed experiments such as Double Chooz ${ }^{2}$ and Daya Bay ${ }^{3}$ are expected to reach. Prior to construction of a neutrino factory, terrestrial experiments may not be able to reach much beyond the 0.01 level.

Figure 6 illustrates some of remaining questions related to masses. We know the solar neutrino mass-squared difference $\delta m_{12}^{2}=m_{2}^{2}-m_{1}^{2}$. In the case of atmospheric neutrinos, the magnitude, but not the sign, of $\delta m_{23}^{2}$ is known. Thus two hierarchies, normal and inverted, are allowed by the data. The absolute scale of neutrino masses, the offset from zero shown in Figure 6, is limited by laboratory experiments (tritium $\beta$-decay) to $\lesssim 2.2 \mathrm{eV}$ (Bonn et al. 1999; Lobashev et al. 1999). The behavior of the neutrino mass under particle-antiparticle conjugation is not known. Extensions of the SM would allow both Dirac and lepton-number-violating Majorana terms, and indeed the presence of both is exploited in the seesaw mechanism to explain the smallness of neutrino mass relative to other SM fermions,

$$
m_{v} \sim m_{\mathrm{D}}\left[\frac{m_{\mathrm{D}}}{M_{\mathrm{R}}}\right]
$$

where $m_{\mathrm{D}}$ is a typical SM Dirac mass and $M_{\mathrm{R}}$ a heavy right-handed neutrino mass. That is, $m_{v}$ is

\footnotetext{
2 http://doublechhoz.in2p3.fr/ and

hep-ex/0606025

${ }^{3}$ http://dayawane.ihep.ac.cn/ and hep-ex/0701029
}

suppressed relative to other SM masses by the small parameter $m_{\mathrm{D}} / M_{\mathrm{R}}$. Indeed, an $M_{\mathrm{R}} \sim 0.3 \times 10^{15}$, near the GUT scale, is suggested by the identification of $m_{3} \sim\left(\delta m_{23}^{2}\right)^{1 / 2} \sim 0.05 \mathrm{eV}$.

Progress will be made on each of these questions in the next decade. Next-generation long-baseline neutrino experiments have, as one of their goals, the use of matter effects to distinguish between the normal and inverted hierarchies ${ }^{4}$. The new tritium $\beta$-decay experiment KATRIN has the potential to tighten the limit on $m_{v_{\mathrm{e}}}$, and thus the absolute scale of neutrino mass, by almost an order of magnitude ${ }^{5}$. Similarly, cosmological analyses that calculate the effects of massive neutrinos on large-scale structure, as deduced from galaxy surveys and from temperature fluctuations in the cosmic microwave background (CMB), place a constraint on the neutrino mass contribution to the closure density. Currently this yields

$$
\sum_{i} m_{v}(i) \lesssim 0.7 \mathrm{eV}
$$

though this limit is expected to tighten significantly when Planck and other future CMB observatories produce data.

Several next-generation neutrinoless double $\beta$-decay experiments are under development, with the ultimate goal of reaching Majorana mass sensitives of

$$
\left|\sum_{i=1}^{2 n} \lambda_{i} U_{e i}^{2} m_{i}\right| \lesssim 0.05 \mathrm{eV}
$$

the scale we noted was set by $\left(\delta m_{23}^{2}\right)^{1 / 2}$. Here the sum extends over neutrino generations, with each neutrino mass eigenstate $i$ contributing in proportion to its mass and to its coupling probability to the electron $U_{e i}^{2}$. Each term is weighted by a phase $\lambda_{i}$ that, if CP is conserved, corresponds to $\pm i$. Thus mass eigenstates will tend to cancel in this sum if they have opposite CP. If CP is violated, the $\lambda_{i}$ also include the effects of two Majorana phases that will be difficult to constrain by other means. Double $\beta$-decay is our most powerful probe of lepton number violation and Majorana masses, and has potentially the most reach as a laboratory test of neutrino mass. It can distinguish among competing neutrino mass scenarios, for example quasi-degenerate schemes versus schemes where the electron neutrino is quite light, though the mass defined by (7) is not simply related to the kinematic mass measured in experiments like tritium $\beta$-decay.

Denoting the mass eigenstates by $v_{1}, v_{2}$ and $v_{3}$, the relationship between the mass and flavor eigenstates is a product of rotations in the 2-3 (atmospheric neutrino), 1-3 (reactor $\bar{v}_{\mathrm{e}}$ disappearance), and 1-2 (solar neutrino)

\footnotetext{
${ }^{4}$ http: //www. fnal.gov/pub/directorate/steering/

5 http: //www-ik.fzk.de/tritium/overview/

index.html
} 
subspaces. Defining the respective angles by $\theta_{23}, \theta_{13}$, and $\theta_{12}$, with $\cos \theta_{\mathrm{X}} \equiv c_{\mathrm{X}}$ and $\sin \theta_{\mathrm{x}} \equiv s_{\mathrm{X}}$, the mixing matrix is

$$
\begin{aligned}
& \left(\begin{array}{c}
v_{\mathrm{e}} \\
v_{\mu} \\
v_{\tau}
\end{array}\right)
\end{aligned}
$$

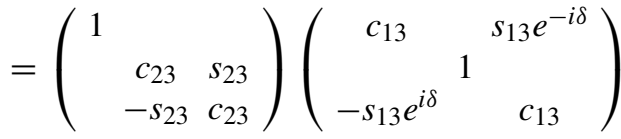

$$
\begin{aligned}
& \times\left(\begin{array}{ccc}
c_{12} & s_{12} & \\
-s_{12} & c_{12} & \\
& & 1
\end{array}\right)\left(\begin{array}{c}
v_{1} \\
e^{i \phi_{2}} \nu_{2} \\
e^{i \phi_{3}} v_{3}
\end{array}\right) \\
& =\left(\begin{array}{ccc}
c_{12} c_{13} & s_{12} c_{13} & s_{13} e^{-i \delta} \\
-s_{12} c_{23} & c_{12} c_{23} & \\
-c_{12} s_{23} s_{13} e^{i \delta} & -s_{12} s_{23} s_{13} e^{i \delta} & s_{23} c_{13} \\
s_{12} s_{23} & -c_{12} s_{23} & \\
-c_{12} c_{23} s_{13} e^{i \delta} & -s_{12} c_{23} s_{13} e^{i \delta} & c_{23} c_{13}
\end{array}\right) \\
& \times\left(\begin{array}{c}
v_{1} \\
e^{i \phi_{2}} v_{2} \\
e^{i \phi_{3}} v_{3}
\end{array}\right) \text {. }
\end{aligned}
$$

This matrix includes three $\mathrm{CP}$-violating phases, the Dirac phase $\delta$ and two Majorana phases $\phi_{2}$ and $\phi_{3}$.

The atmospheric and solar neutrino experiments have determined $\theta_{23} \sim 45^{\circ}$ and $\theta_{12} \sim 30^{\circ}$ and, while greater accuracy is always important (especially to determine how close $\theta_{23}$ might be to the maximal mixing limit of $\pi / 4$ ), most attention is now focused on determining the unknown parameters in the mixing matrix. Perhaps most important is the third mixing angle $\theta_{13}$, which, as noted earlier, is so far only bounded by reactor neutrino results. New reactor experiments currently in preparation, Double Chooz and Daya Bay, are designed to reach sensitivities of approximately 0.02 and 0.008 , respectively.

Given that the source of $\mathrm{CP}$ violation responsible for the excess of matter over antimatter in our universe is still uncertain, the determination of the scale of leptonic $\mathrm{CP}$ violation is also a major goal. With suitable attention to matter effects associated with neutrino beams passing through the earth and to other parameter degeneracies (Barger, Marfatia \& Whisnat 2002), CP violation can be determined in long-baseline neutrino oscillation experiments that compare $P\left[v_{\mu} \rightarrow v_{\tau}\right]$ with $P\left[\bar{v}_{\mu} \rightarrow \bar{v}_{\tau}\right]$. The $\mathrm{CP}$ violation is proportional to the Jarlskog invariant:

$$
\sin \left(2 \theta_{12}\right) \sin \left(2 \theta_{23}\right) \sin \left(2 \theta_{13}\right) \cos \left(\theta_{13}\right) \sin (\delta) .
$$

The first two factors are known to be large. Thus a demonstration that $\theta_{13}$ is not too small would imply significant sensitivity to $\mathrm{CP}$ violation. Probing such $\mathrm{CP}$ violation is one of the major goals for very long baseline neutrino oscillation experiments, where the relative size of the CPviolating observable grows with distance (Barger et al. 2007).

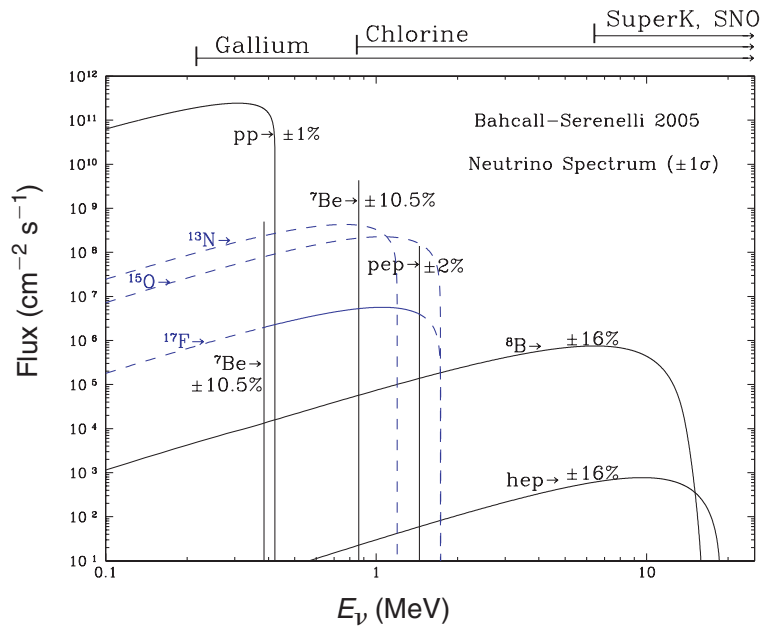

Figure 7 The spectrum of solar neutrinos, from Bahcall ${ }^{6}$. SNO and Super-Kamiokande spectral measurements were limited to neutrinos with energies above $5 \mathrm{MeV}$. Borexino will make the first direct measurement of sub-MeV neutrinos. The $\mathrm{CNO}$ fluxes could be identified in an experiment like $\mathrm{SNO}+$.

\subsection{Low-Energy Neutrino Spectroscopy}

Pending results from Borexino and KamLAND on their efforts to measure ${ }^{7} \mathrm{Be}$ neutrinos, direct real-time measurements are so far limited to the ${ }^{8} \mathrm{~B} v$ spectrum above $5 \mathrm{MeV}$. Thus $99.99 \%$ of the flux has not been detected by direct means, including the pp/pep and ${ }^{7} \mathrm{Be}$ neutrinos that tag the ppI and ppII cycles (see Figure 7, taken from Bahcall $\left.^{6}\right)$.

The oscillation parameters deduced from global fits to solar neutrino data indicate that the solar $v_{\mathrm{e}}$ spectrum will be distorted by matter effects. Yet so far there has been no direct measurement of the energy dependence in the survival probability $P\left(E_{v}\right)$ or of MSW day-night effects due to neutrino passage through the earth. The oscillation parameters indicate that the level-crossing boundary of the 1-2 MSW triangle will be encountered at a neutrino energy of $\sim 3 \mathrm{MeV}$. Thus one can explore the transition from vacuum oscillations to matter oscillations by mapping $P\left(E_{v}\right)$ from low to high neutrino energies. Borexino, now operating, will determine $P\left(E_{v}=0.86 \mathrm{MeV}\right)$, for example.

A variety of $\mathrm{CC}$ and $\mathrm{NC}$ pp-neutrino detectors are under development. In addition to the exploration of matter effects, these detectors will be able to exploit nature's most intense and well-characterised source of electron neutrinos: the solar pp flux and spectrum are known to an accuracy of about $1 \%$. While experiments like KamLAND have succeeded in reducing uncertainties on $\delta m_{12}^{2}$ by measuring $P\left(\bar{v}_{\mathrm{e}}\right)$ at different baselines, uncertainties in the reactor $\bar{v}_{\mathrm{e}}$ spectrum limit the accuracy of $\theta_{12}$ determinations. But solar pp-neutrino measurements could, in principle, determine this angle to $\sim 1 \%$.

\footnotetext{
${ }^{6}$ http: //www.sns.ias.edu/jnb/
} 


\subsection{Testing Stellar Modeling: The CNO Neutrinos}

Despite the minor role CNO neutrinos play in our Sun, there is strong motivation for exploiting these neutrinos as a quantitative test of our understanding of the $\mathrm{CNO}$ cycle:

- The CNO cycle, due to its sharper dependence on stellar core temperature, is the reaction chain that sustains massive main-sequence stellar evolution. This dependence is reflected in the corresponding neutrino fluxes, which vary as $\phi(\mathrm{CNO}) \sim T_{\mathrm{C}}^{20}$. Because SNO and Super-Kamiokande have made accurate measurements of the most temperature-dependent component of the pp-chain $\left(\phi\left({ }^{8} \mathrm{~B}\right) \sim T_{\mathrm{C}}^{24}\right)$, we know the core temperature in our Sun to an accuracy of about $1 \%$. Thus we can now use the solar core as a calibrated laboratory in which to test our understanding of the $\mathrm{CNO}$ cycle.

- The CNO cycle is important to other systems of current interest, such as the first massive metal-poor stars (where hydrogen burning via the $\mathrm{CNO}$ cycle turns on only after an early phase of $3 \alpha \rightarrow{ }^{12} \mathrm{C}$ produces metals).

- One of the principal assumptions of the SSM is the identification of today's surface metal abundances with the zero-age core metallicity. A measurement of CNO neutrinos is a direct check on this assumption, as the flux is proportional to core metallicity.

- This issue, using surface observations to constrain the core, is now central to the principal anomaly in the SSM, that new surface metal determinations have placed helioseismology results (e.g. deduced sound speeds, estimates of the depth of the convective zone) in conflict with SSM predictions (Asplund, Grevesse \& Sauval 2006).

- The CNO cycle is thought to be responsible for an early convective stage in our Sun, extending about $10^{8} \mathrm{yr}$, driven by out-of-equilibrium burning. Current efforts to build 2D/3D extensions of the SSM are an important step toward modeling the early Sun. Thus one would like to verify that equilibrium CNO-burning is understood, to establish a foundation for later studies of solar convection and out-of-equilibrium burning.

- The nuclear physics of the CNO cycle has been put on much firmer ground due to recent measurements of the controlling cross section.

The sharp $T_{\mathrm{C}}$-dependence of CNO hydrogen burning is important to the stability of massive stars. While a minor contributor to SSM energy production $(\sim 1 \%)$, the $\mathrm{CNO}$ cycle does produce measurable solar neutrino fluxes (Bahcall \& Pinsonneault 2004):

$$
\begin{aligned}
{ }^{13} \mathrm{~N} & \rightarrow{ }^{13} \mathrm{C}+e^{+}+v_{\mathrm{e}}, \quad E_{\max }=1.199 \mathrm{MeV}, \\
\phi & \sim 5.7 \times 10^{8} \mathrm{~cm}^{-2} \mathrm{~s}^{-1} \\
{ }^{15} \mathrm{O} & \rightarrow{ }^{15} \mathrm{~N}+e^{+}+v_{\mathrm{e}}, \quad E_{\max }=1.732 \mathrm{MeV}, \\
\phi & \sim 5.0 \times 10^{8} \mathrm{~cm}^{-2} \mathrm{~s}^{-1} .
\end{aligned}
$$

To predict the response of terrestrial detectors to these sources one needs $T_{\mathrm{C}}$ (calibrated in the ${ }^{8} \mathrm{~B}$ flux

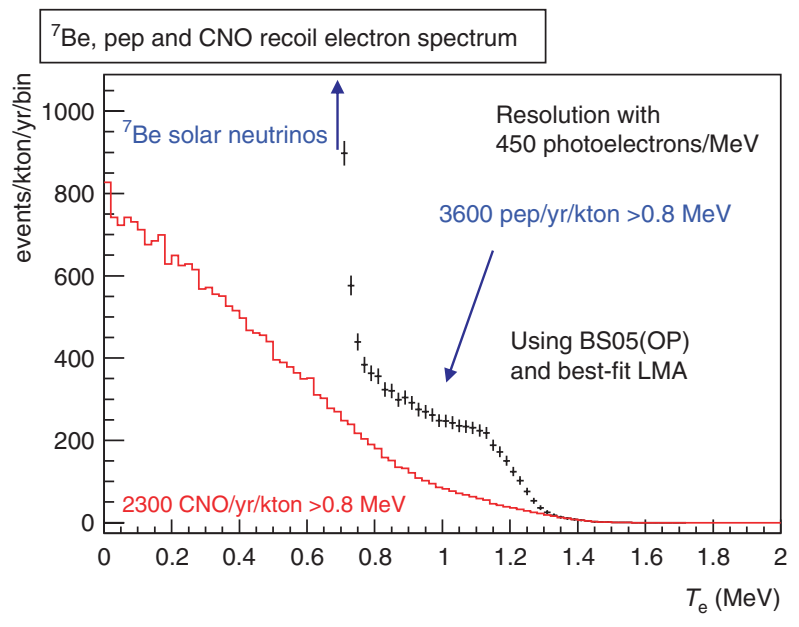

Figure 8 The recoil spectrum expected from elastic scattering of pep, ${ }^{7} \mathrm{Be}$, and $\mathrm{CNO}$ neutrinos in $\mathrm{SNO}+$. Figure from M. Chen ${ }^{7}$ (Chen 2005).

measurements by SNO and Super-Kamiokande), the oscillation parameters $\delta m_{12}^{2}$ and $\theta_{12}$, and the nuclear cross sections for the CNO cycle. Recent progress has also been made on nuclear physics: new measurements by the LUNA collaboration (Lemut et al. 2006) and at TUNL (Runkle et al. 2005) have reduced uncertainties in the rate-controlling ${ }^{14} \mathrm{~N}(\mathrm{p}, \gamma)$ cross section. LUNA measured the $S$-factor down to $70 \mathrm{keV}$, finding a result that is $50 \%$ smaller than the previous 'best value'. This revision has had a significant impact on stellar age determinations, pushing back globular cluster ages by an estimated $0.7-1.0$ Gyr.

There is a new idea for building a high-counting-rate detector sensitive to $\mathrm{CNO}$ neutrinos, construction of a large-volume scintillation detector in the cavity previously occupied by SNO. The new detector, SNO+ (Chen 2005), if developed for solar neutrinos (in addition to double $\beta$-decay), would be able to detect about $2300 \mathrm{CNO}$ neutrinos per year per 1000 ton, above a threshold of about $0.8 \mathrm{MeV}$, as shown in Figure 8. It would appear that a flux measurement accurate to $\sim 10 \%$ might be possible.

The combination of a practical experiment, more certain nuclear physics, a calibrated solar core temperature, and known neutrino parameters $\delta m_{12}$ and $\theta_{12}$ appear to make a direct measurement of core metallicity possible. The new surface abundances that have been derived from improved 3D atmospheric solar absorption line analyses are difficult to dismiss, despite the tension they have generated between the SSM (and its neutrino predictions) and helioseismology. The new abundances generally bring the Sun into better accord with galactic composition trends (Turck-Chieze et al. 2004). It may turn out that the SSM assumption that equates surface and zero-age core metallicity is unjustified, that some evolutionary effect breaks this equivalence. In any case, the opportunity to test this assumption experimentally should be taken.

\footnotetext{
${ }^{7}$ http://snoplus.phy.queensu.ca/
} 


\section{Summary}

Neutrino astrophysics and the theories of the origin of the elements, the main theme of this conference, share a common history. Laboratory astrophysics has made solar neutrino physics into a quantitative field, and allowed experimenters to anticipate the kinds of major discoveries that justified experiments like SNO and SuperKamiokande. The results - discovery of neutrino mass and flavor mixing characterized by large angles — are of great importance, providing our first constraints on physics beyond the SM of particle physics. But as summarised here, the list of remaining laboratory neutrino physics questions is long. The answers to the open questions will be important in helping us characterise extreme astrophysical and cosmological neutrino environments. The needed 20-year program of laboratory and astrophysical neutrino studies is not unlike the laboratoryastrophysics interface that Willie Fowler cultivated to help us understand the origin of the elements.

Despite the current focus on particle physics properties of neutrinos, solar neutrino spectroscopy remains an important probe of the SSM and stellar evolution. The arguments for measuring the CNO flux, using our Sun as a calibrated laboratory, seem particularly strong. Such a program would effectively test our understanding of the hydrogen burning mechanism for massive main-sequence stars. It would also address the primary discrepancy in the SSM, the tension between helioseismology and neutrino flux predictions that follows from new analyses of surface metallicity.

\section{References}

Abdurashitov, J. N. et al., 1999a, PhRvL, 83, 4686

Abdurashitov, J. N. et al., 1999b, PhRvC, 60, 055801

Adelberger, E. G. \& Haxton, W. C., 1987, PhRvC, 36, 879

Ahmad, Q. R. et al., 2002, PhRvL, 89, 011301

Altmann, M. et al., 2000, PhLB, 490, 16

Alvarez, L. W., 1949, Univ. California Radiation Laboratory Report UCRL-328

Apollonio, M. et al., 1999, PhLB, 466, 415
Arpesella, C. et al., 2007, PhLB, submitted (arXiv:0708.2251v2)

Asplund, M., Grevesse, N. \& Sauval, J., 2006, NuPhA, 777, 1

Bahcall, J. N., 1964, PhRvL, 12, 300

Bahcall, J. N. \& Barnes, C. A., 1964, PhL, 12, 48

Bahcall, J. N. \& Davis, R., Jr., 1982, in Essays in Nuclear Astrophysics, Ed. Fowler, W. A. (Knudsen), 243

Bahcall, J. N. \& Pinsonneault, M. H., 2004, PhRvL, 92, 121301

Bahcall, J. N., Bahcall, N. A. \& Shaviv, G., 1968, PhRvL, 20, 1209

Barger, V. et al., 2007, arXiv:0705.4396v1

Barger, V., Marfatia, D. \& Whisnant, K., 2002, PhRvD, 65, 073023

Bethe, H. A., 1986, PhRvL, 56, 1305

Bonetti, R. et al., 1999, PhRvL, 82, 5205

Bonn, J. et al., 2001, PhLB, 91, 273

Burbidge, E. M., Burbidge, G. R., Fowler, W. A. \& Hoyle, F., 1957, RvMP, 29, 547

Cameron, A. G. W., Stellar Evolution, Nuclear Astrophysics, and Nucleogenesis, Atomic Energy of Canada Report, CRL-41

Castellani, V., Degl'Innocenti, S., Fiorentini, G., Lissia, M. \& Ricci, B., 1994, PhRvD, 50, 4749

Chen, M. C., 2005, NuPhS, 145, 65

Davis, R., Jr., 1955, PhRv, 97, 766

Davis, R., Jr., 1964, PhRvL, 12, 303

Davis, R., Jr., Harmer, D. S. \& Hoffman, K. C., 1968, PhRvL, 20 1205

Eguchi, K. et al., 2003, PhRvL, 90, 021802

Hampel, W. et al., 1999, PhLB, 447, 127

Hardy, J. C. \& Verrall, R. I., PhRvL, 13, 764

Haxton, W. C., 1986, PhRvL, 57, 1275

Haxton, W. C., Parker, P. D. \& Rolfs, C. E., 2006, NuPhA, 777, 226

Hayashi, C., 1966, ARA\&A, 4, 171

Hirata, K. S. et al., 1991, PhRvD, 44, 2241

Holmgren, H. D. \& Johnston, R. L., 1959, PhRv, 113, 1556

Hosaka, J. et al., 2006, PhRvD, 73, 112001

Junghans, A. R. et al., 2003, PhRvC, 68, 065803

Lemut, A. et al., 2006, PhLB, 634, 483

Lobashev, V. M. et al., 1999, PhLB, 460, 227

Mikheyev, S. P. \& Smirnov, A. Yu., 1986, NCimC, 9, 17

Parke, S. J., 1986, PhRvL, 57, 1275

Pontecorvo, B., 1946, Chalk River Laboratory Report PD-205; and 1968, SvPhJ, 26, 984

Reeder, P. L., Poskanzer, A. M. \& Esterlund, R. A., 1964, PhRvL, 13,767

Runkle, R. C., Champagne, A. E., Angulo, C., Fox, C., Iliadis, C., Longland, R. \& Pollanen, J., 2005, PhRvL, 94, 082503

Turck-Chieze, S., Couvidat, S., Piau, L., Ferguson, J., Lambert, P., Ballot, J., García, R. A. \& Nghiem, P., 2004, PhRvL, 93, 211102 Wolfenstein, L., 1978, PhRvD, 17, 1 